\title{
Catalogs of Wide Binaries: Impact on Galactic Astronomy
}

\author{
Julio Chanamé \\ Space Telescope Science Institute, Baltimore, MD, USA \\ email: jchaname@stsci.edu
}

\begin{abstract}
Wide binaries, particularly in large numbers and as free from selection biases as possible, constitute a largely overlooked tool for studying the Galaxy. The goal of this review is to highlight the potential inherent to large samples of field wide binaries for research on problems as varied as star formation in the early Galaxy, the nature of halo dark matter, the evolution of the stellar halo, new geometric distances, metallicities, masses, and ages of field stars and white dwarfs, and much more. Using the Revised NLTT as an illustrative example, I review the main steps in the assembly of a large catalog of wide binaries useful for multiple applications. The capability of cleanly separating between the Galactic disk and halo populations using good colors and proper motions is emphasized. The critical role of large surveys for research on wide binaries as well as for the better understanding of the Galaxy in general is stressed throughout. Finally, I point out the potential for assembling new samples of wide binaries from available proper-motion surveys, and report on current efforts of using the SDSS towards this goal.
\end{abstract}

Keywords. surveys, catalogs, (stars:) binaries: general, stars: fundamental parameters, stars: kinematics, Galaxy: general, (cosmology:) dark matter

\section{Introduction}

Knowledge obtained from the study of stars in binary systems has been fundamental for astronomy, and influences almost all of its branches. Their impact is easily illustrated with just a few examples: the masses of individual stars (the single most important parameter in stellar evolution) are measured with confidence only for stars in binary systems $\dagger$; eclipsing binaries provide a primary method for measuring distances (Paczyński 1997) and are playing a major role in securing the base of the cosmological distance ladder (Ribas et al. 2005; Bonanos et al. 2006); close binary systems harboring white dwarfs, as likely progenitors of type-Ia supernovae, are thought to be main players driving the chemical evolution of galaxies and the intergalactic medium (Pagel 1997). And the list can go on and on.

Most of that knowledge, however, has come from the study of binary systems in close orbits, while the population of binaries at the wide end of the distribution of orbital separations (semimajor axes $a \gtrsim 100 \mathrm{AU}$ ) remains poorly explored. Clearly, the main reason behind this historical bias is the much longer orbital timescale inherent to very wide binary systems (of the order of hundreds of years and longer), making their unambiguous identification a task that also requires a relatively long timescale (though, fortunately, not as long as their complete periods!). In contrast to the case of close binaries, where some kind of variability is typically the property that helps uncover them, a reasonably large number of very wide binaries can not be identified via the typical photometric or

$\dagger$ Stellar mass measurements for single stars have been achieved thanks to gravitational microlensing as well (Drake et al. 2004; Jiang et al. 2004). However, the errors in this technique are not yet competitive with those for stars in binary systems. 
spectroscopic campaign lasting from several months to a few years. Instead, wide binaries require accurate astrometry over timescales of the order of decades.

Nevertheless, wide binaries hold great potential for a large variety of studies in Galactic astronomy. A summary list of today's applications of wide binaries can include, in no particular order:

- probing for the processes and conditions of star formation as a function of age and metallicity in star-forming regions (White \& Ghez 2001), and as a function of environment (disk vs. halo) during the assembly of the Galaxy (Chanamé \& Gould 2004);

- to obtain new and accurate distances to faint low-mass stars in the field (Gould \& Chanamé 2004; Lépine \& Bongiorno 2006), allowing high-precision studies of stellar properties near the bottom of the main sequence (Patten et al. 2006);

- determination of the metallicities of field M-dwarfs (Bonfils et al. 2005) and the study of the age-metallicity relation in the Galactic disk (Monteiro et al. 2006);

- tests of not well constrained stellar evolutionary processes such as internal mixing and diffusion via the study of the surface abundances of the members of wide binaries with twin components (Martín et al. 2002);

- exploration of the age and chemical evolution of the stellar halo and its underlying substructure via the study of halo wide binaries with evolved components (Ivans, Chanamé, \& Gould, in preparation);

- measurement of the masses of white dwarfs and constraints on the (dark) mass density of stellar remnants in the local Galactic disk (Silvestri et al. 2001);

- to place severe constraints on the nature of halo dark matter that complement the decades-long microlensing campaigns (Yoo, Chanamé, \& Gould 2004), and to explore the dynamical and merger history of the Galaxy (Allen \& Poveda 2007);

- studies of the initial-to-final mass relation of white dwarfs via the determination of the mass of their main-sequence progenitors (Catalán et al. 2007; Silvestri et al. 2005);

- constraining mass loss during post main sequence stages, white dwarf progenitor masses, and the density of matter returned to the interstellar medium via the investigation of the evolution of orbital separations of wide binaries harboring evolved components (Johnston, Oswalt, \& Valls-Gabaud 2007);

- studying the impact of planetary systems on the evolution of their host stars via the detailed surface abundances of stars in wide binaries (Desidera et al. 2004).

The main purpose of this review is therefore to highlight the large potential inherent to large samples of wide binariest for the study of the Galaxy. The emphasis on the words large samples is not accidental: various of the applications mentioned above are not necessarily the product of studies of wide binaries on a system by system basis but, rather, statistically as a population. They have only been possible thanks to the relatively large numbers of genuine binaries that became available when astronomers changed their strategies and started to look for them in a systematic way, making big efforts to avoid possible selection biases. This change of approach, in turn, only became possible with the construction and exploitation of large photometric and astrometric databases such as the NLTT, USNO, Hipparcos, 2MASS, etc. Therefore, the critical role of large surveys in this revolution, not only in the field of wide binaries but for Galactic astronomy in general, cannot be emphasized enough, and this crucial point is stressed again in $\S 2$ and throughout the present contribution.

In $\S 3$ a brief report is presented of the status of the currently available catalogs of wide binaries, using the case of the Revised NLTT (rNLTT; Chanamé \& Gould 2004) catalog

$\dagger$ The discussion in the present contribution is restricted to wide binaries in the field, i.e., does not include systems in clusters and/or star forming-regions. 
to illustrate the steps involved in their construction. A description of the ongoing efforts and prospects of using the Sloan Digital Sky Survey (SDSS) to build an even larger catalog of wide binaries is presented in $\S 3.2$. Finally, a few of the various applications of catalogs of wide binaries to problems of Galactic astronomy are briefly outlined in $\S 4$.

\section{Surveys: the crucial starting point}

Progress in astronomy over the last 25 or so years has been deeply influenced by the steady increase in importance of the role of large and systematic surveys of all types. Several of these even prompted the deployment of instrumentation beyond Earth's atmosphere. The impact of these strategies is now seen across areas that range from the cosmological (HST, 2dF, WMAP, ...) to that of extrasolar planets (SuperWasp, Kepler). In the Galactic context, the extraordinary explosion of new science and discoveries that the SDSS (a survey mainly designed to address cosmological questions) is allowing at this very moment has its most immediate predecessors, both scientific as well as technological, in surveys such as Hipparcos and 2MASS. Today, the case for staying on the same track is evidenced by the efforts (and funding!) put in upcoming surveys such as GAIA.

Research on wide binaries is no exception to this trend and progress in this area is also intimately tied to the development and use of large databases. Commonly referred to as common proper-motion systems, a large number of wide binaries is a natural and relatively straightforward product of astrometric campaigns and proper-motion surveys.

Moreover, the combination of good photometry with good astrometry, either coming from a single survey or from the combination of separate ones, has allowed in the last few years the clean separation between large numbers of stars unambiguously belonging to the disk and halo of the Galaxy, thus opening a new avenue for studying Galactic structure and evolution with high statistical significance. On the subject of our interest here, the rNLTT catalog of disk and halo wide binaries, assembled from the combination of surveys such as the NLTT, USNO-A, Hipparcos, and 2MASS, constitutes an excellent example of this synergy, and its capacity of telling whether a single star belongs to the disk or the halo of the Galaxy constitutes a powerful tool for the unambiguous identification of pairs of stars that, even though having proper motions sharing the same magnitude and direction on the sky, are not really bound to each other, and would contaminate a sample of wide binaries selected only on common proper-motion criteria (see $\S 3.1$ ).

The big advantage of the strategy of surveys lies not only in the greatly improved statistics but, most importantly, in the degree of completeness of the resulting databases, which, with well understood selection effects, if any, permits a relatively unbiased view of whatever the particular survey aims at. It is this crucial characteristic that, in the case of wide binaries, makes possible two of their most interesting applications to astronomy, as are their use as probes of the nature of dark matter and of the processes and conditions of star formation (see $\S 4$ ).

The approach of large surveys has proven to be of great power and is likely to stay as one of the favored ways to advance in astrophysics. People interested in wide binaries and their applications must therefore consider carefully what can be done with the already existing databases as well as prepare in advance for an efficient use and exploitation of the surveys to come. Joint Discussion 13 on this very same IAU General Assembly, entitled Exploiting Large Surveys for Galactic Astronomy, dealt with this subject in detail, and readers are encouraged to consult those proceedings (see also their website at http://clavius.as.arizona.edu/vo/jd13/). 


\section{Catalogs of field wide binaries}

As noted before, being the characteristic fingerprint of wide binaries the almost identical proper-motion vectors of their two stars (up to differences due to measurement uncertainty and relative orbital motion only), they are intimately associated to propermotion surveys. The rNLTT wide binaries, extracted from the Luyten survey of high proper-motion stars (Luyten 1979, 1980), constitute likely the most homogeneous and complete catalog of such objects available today, although not the only one.

Before the rNLTT, the most successful efforts to assemble large numbers of wide binaries were those of Poveda et al. (1994) and Allen et al. (2000), who based their searches on databases such as Gliese's Catalog of Nearby Stars and the NLTT itself, though not exploiting the latter in its full capacity. See the contributions by A. Poveda and C. Allen on these proceedings for details on their samples and some of their uses in astronomy. The efforts by Allen et al. (2000) and Zapatero Osorio \& Martín (2004) concentrated on the search for wide companions to metal-poor stars selected from databases such as the Carney-Latham surveys. A few earlier attempts to identify smaller samples of field wide binaries are reported in the introductory section of Chanamé \& Gould (2004).

Other proper-motion catalogs available today that hold potential for a search of wide binaries include UCAC (Rafferty et al. 2001), SuperCOSMOS (Hambly et al. 2001), LSPM (Lépine \& Shara 2005), and SDSS $\cap$ USNO-B (Gould \& Kollmeier 2004; Munn et al. 2004). Although a few new common proper-motion pairs of intrinsic interest have been identified from these databases (Scholz et al. 2002, 2005; Seifahrt et al. 2005; Monteiro et al. 2006), only one report of a systematic search for wide binaries using these catalogs exists, which appeared the very same day this contribution was to be submitted. In a manner analogous to the search of Gould \& Chanamé (2004) in the rNLTT, Lépine \& Bongiorno (2006) searched in the LSPM catalog for faint common proper-motion companions of Hipparcos stars, uncovering 521 systems, of which 130 are new (see $\S 4$ ).

The optimal construction of a catalog of field wide binaries is outlined in $\S 3.1$, using the case of the rNLTT as an illustrative example. It will be seen how, with appropriate photometric and astrometric data, it is possible to not only identify genuine field wide binaries, but also to determine with high confidence, on a pair by pair basis, their membership in either the disk or the halo populations of the Galaxy. Disentangling between pairs belonging to the Galactic thin and thick disks, however, would require more information, such as the full three-dimensional velocities and metallicities of the stars. Finally, in $\S 3.2$, I describe an ongoing search for wide binaries in SDSS $\cap$ USNO-B.

\subsection{Building a catalog: the rNLTT wide binaries}

The NLTT proper-motion survey was available long before the Chanamé \& Gould (2004) work, and Luyten himself identified and recorded a substantial fraction of the NLTT wide binaries in his Luyten Double-Star Catalog (LDS, Luyten 1940-87). However, two factors worked against the construction of a complete sample of wide binaries from the original NLTT. First, the crude photographic colors did not allow the construction of a reliable reduced proper motion (RPM) diagram, thus preventing the identification of chance alignments (i.e., pairs of unrelated stars whose proper-motion vectors are aligned on the sky just by chance) via the non-consistent colors of the stars in such pairs (more below). Second, the large proper-motion errors in the original NLTT did not allow Luyten to identify a fair number of genuine binaries at large angular separations (the non-Luyten binaries of Chanamé \& Gould 2004) because it was impossible to separate them from the numerous unrelated optical pairs at those separations.

Only with the construction of the Revised NLTT (Gould \& Salim 2003; Salim \& Gould 2003) could these problems be overcome. An upgraded version of the original NLTT, 

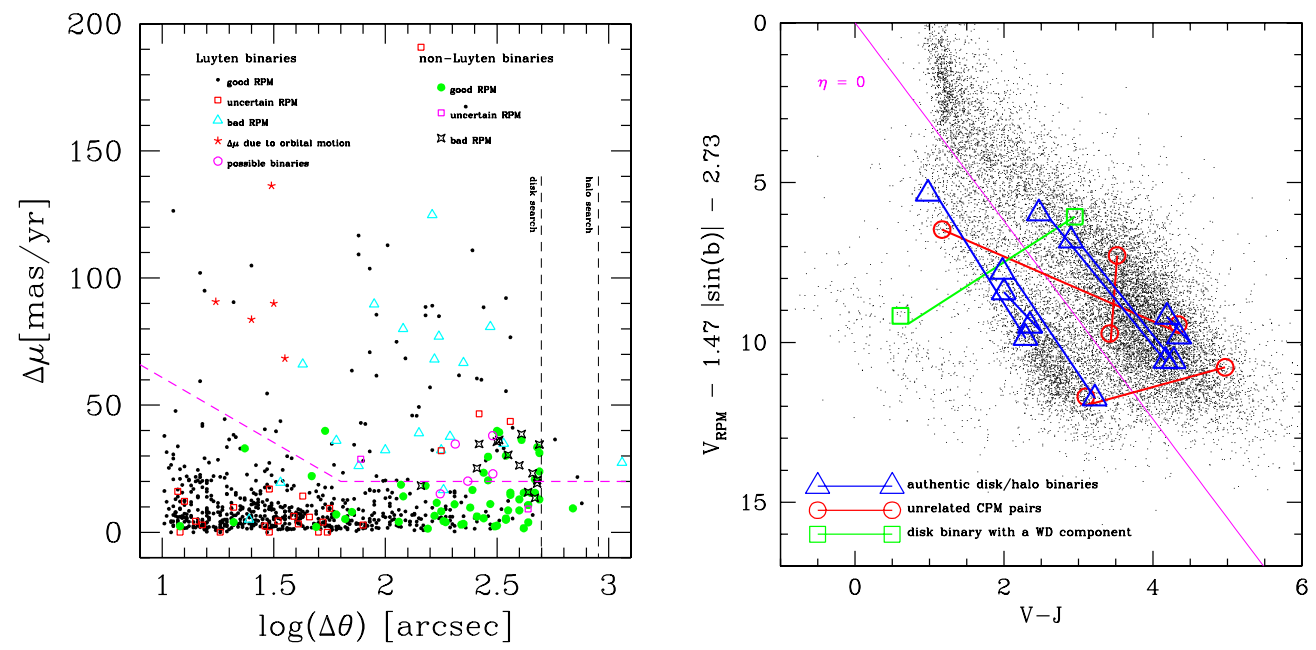

Figure 1. (a; left) Initial selection of candidate binaries in rNLTT: vector proper motion difference vs. angular separation for systems with $\Delta \theta>10^{\prime \prime}$. Pairs below the dashed line were automatically accepted as candidate binaries for their subsequent classification. The dashed vertical lines indicate the upper limits of the homogeneous search for disk and halo binaries. (b; right) Reduced proper motion diagram of rNLTT stars, clearly separating the disk, halo, and white dwarf tracks. A few examples of how this diagram is used to test and classify binaries are shown (see text).

the rNLTT catalog provides optical and (CCD) infrared photometry for the majority of the NLTT stars by matching them to the USNO-A and 2MASS surveys. Thanks to the better colors and large color baseline, this proved sufficient to permit a clean separation between disk and halo stars using a RPM diagram (Salim \& Gould 2002). Furthermore, the better temporal baseline of the rNLTT relative to the NLTT translated into a substantial improvement in the accuracy of the proper-motion measurements, which allowed Chanamé \& Gould (2004) to perform a uniform and complete search of wide common proper-motion pairs up to angular separations much larger than what Luyten was able to do. It is worth noticing, here again, the fundamental importance of large surveys and the potential inherent in their combination and adequate exploitation.

Still, with the main shortcomings of the original NLTT overcome with the publication of the rNLTT, it was by no means straightforward to dig out a uniformly selected catalog of wide binaries from it. In order to produce a sample of wide binaries as free from selection effects as possible, a number of complications had to be dealt with, such as blending, saturation, missing independent proper-motion measurements, and others. See Chanamé \& Gould (2004) for a detailed account of the solutions to these problems. Here only a brief outline of the selection and classification of the rNLTT wide binaries is given.

The first step is to select all pairs of stars that seem to be moving together on the sky, i.e., all pairs with similar proper-motion vectors. This was done by plotting the magnitude of the vector proper motion difference (denoted by $\Delta \mu=|\Delta \boldsymbol{\mu}|$ ) of the components of these pairs against their angular separation $(\Delta \theta)$, as shown in Figure 1a. Pairs with separations smaller than $\Delta \theta=10^{\prime \prime}$ as measured by Luyten were not subjected to this selection because of the small chance of two unassociated stars lying so close in both position and velocity space. Thus the plot in Figure 1a begins at $\Delta \theta=10^{\prime \prime}$ and for pairs with smaller separations all efforts were focused on their classification (which, based on the consistency of their colors, constitutes another test for their reality). 
For the rest of common proper-motion systems wider than $10^{\prime \prime}$, good candidates were considered to be those falling below the dashed line in Figure 1a, set at wide separations at $\Delta \mu=20$ mas yr$^{-1}$. Note that all the NLTT stars are moving faster than 180 mas yr$^{-1}$, so the above maximum- $\Delta \mu$ requirement is already a strong test, and pairs that satisfy it

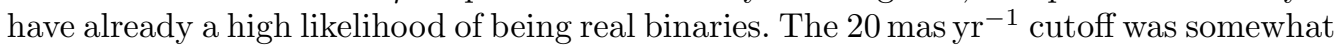
relaxed at closer separations because the chance of contamination by unassociated pairs is lower and also because some real pairs at close separations can have significant orbital motions (pairs indicated by red asterisks in Figure 1a).

This search for rNLTT wide binaries was systematically done up to separations of $\Delta \theta=500^{\prime \prime}$ for disk binaries and $\Delta \theta=900^{\prime \prime}$ for halo binaries, thus avoiding any possible selection effects as a function of angular separation. In the same spirit, the exclusion from the analysis of pairs outside the allowed region of the $\Delta \theta-\Delta \mu$ plane does not mean that all these systems should be regarded as false binaries. Rather, it simply reflects the extreme care put in being as complete and free from selection effects as possible, but at the same time rigorous in not being contaminated by unrelated optical pairs. It is only thanks to this characteristic that the rNLTT wide binaries (or any other catalog similarly constructed) can be used as probes of star formation as well as of the nature of dark matter in the Galaxy.

The second test for the reality of the (up to this point) candidate binaries is related with their classification as either disk or halo pairs. This is done via examination of each pair in a RPM diagram, and all the possibilities are illustrated in Figure 1b, where the clear separation between the tracks of disk and halo stars (at both sides of the line labeled $\eta=0$ ) is evident. When classifying the binaries, one expects not only that both components belong to the same population, but also that their positions on the RPM diagram are consistent with both stars having the same age and metallicity. Therefore, for real binaries (blue triangles in Fig. 1b) one expects that the line connecting their positions on the RPM diagram should be approximately parallel, within measurement errors, to the corresponding disk or halo track. Candidate pairs composed of one disk and one halo member are rejected as chance alignments, and so are pairs composed of two disk or two halo stars if the line connecting them is inconsistent with being parallel to the respective sequence (red circles in Figure 1b). The only cases permitted not to follow this parallel rule are those involving a white dwarf companion (as the green pair of squares in Figure 1b).

The above two-step procedure yielded 999 genuine wide binaries: 883 pairs belonging to the Galactic disk and 116 belonging to the Galactic halo. Among the disk binaries, 82 pairs contained a white dwarf, while no halo stars with clear white dwarf companions were found.

\subsection{Wide binaries from the $S D S S \cap U S N O-B$}

The Sloan Digital Sky Survey, providing superb optical CCD photometry for almost a quarter of the sky, is already revolutionizing our understanding of the Galaxy on several fronts. Although a single-epoch survey, it can be combined with older photometric and/or proper-motion surveys in order to produce the two epochs necessary to compute new proper motions for its stars. Gould \& Kollmeier (2004) and Munn et al. (2004) already accomplished this for the SDSS First Data Release by cross-correlating it with the USNO-B catalog, producing nearly 400,000 stars down to $r^{\prime}<20$, moving faster than

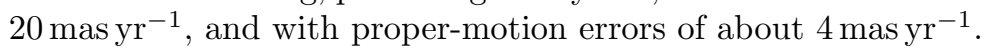

Given the depth and sky coverage of SDSS, there is little doubt that it will provide a significantly larger number of binaries than the rNLTT, and efforts toward this goal are already underway (see Smith et al. 2005 and Greaves 2005 for recent searches in 

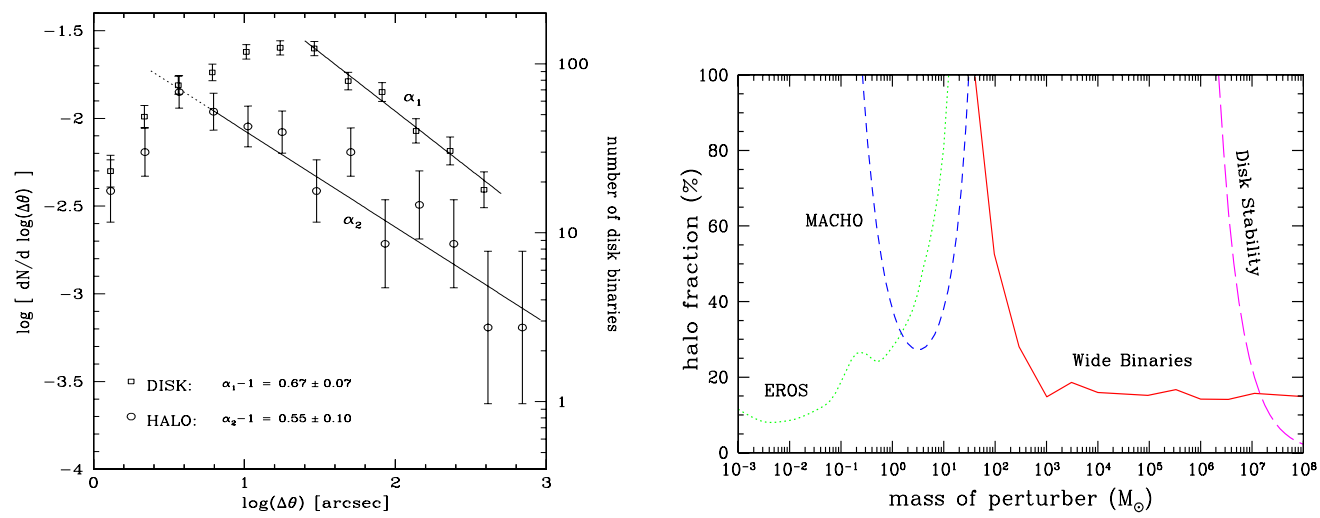

Figure 2. (a; left) Distribution of angular separations for the rNLTT disk and halo wide binaries (Chanamé \& Gould 2004). Note that disk and halo binaries show basically the same distributions, and that the data for both populations of binaries are consistent with a single power-law out to the widest separations, with no hint of a break or cutoff. (b; right) Exclusion contours for MACHO dark matter at the $2 \sigma$ level of the microlensing experiments and the rNLTT halo wide binaries (Yoo, Chanamé, \& Gould 2004).

SDSS of pairs with white dwarf components). The challenge, however, lies in being able to distinguish the truly bound systems from among the large number of spurious pairs inevitably present in such a large collection of stars with very similar proper motions. For the nearby Luyten stars, with proper motions $|\boldsymbol{\mu}|>180$ mas yr $^{-1}$, just the combination of photometry and astrometry was enough to do the selection. But with the more distant SDSS stars, moving at $20-40$ mas yr$^{-1}$, the measured $\Delta \vec{\mu}$ alone does not reject all the apparent binaries, even though the photometry is excellent. Therefore, it is necessary to obtain radial velocities of the components of the common proper-motion candidates that pass the first tests (consistent photometry and proper motions) to clean the initial sample and select the real binaries. Binary candidates have been selected and the radial velocities of the brightest pairs have already been measured using the SMARTS 1.5-m telescope at CTIO (Chanamé, van der Marel, van de Voort, \& Gould, in preparation). The large majority of targets, however, with magnitudes in the range $16<r<20$, require larger apertures. Our plans to complete these observations using telescopes at the MDM and Kitt Peak Observatories are already underway.

\section{Impact of wide binary catalogs on Galactic astronomy}

Here, I only point out a few of the various applications of wide binaries to Galactic astronomy that are outlined in $\S 1$. See T. Oswalt's contribution for the several uses of wide binaries involving white dwarfs, and those of C. Allen and A. Poveda for ways of exploiting their potential as probes of dynamical processes in the Galactic disk.

One of the most useful characteristics of wide binaries is that both components share properties such as age, metallicity, distance, etc., and, therefore, when any of these is measured for one of them, typically the primary, it automatically can be assigned to the fainter secondary. This has been exploited to obtain parallaxes to faint low-mass stars in the field by Gould \& Chanamé (2004) and Lépine \& Bongiorno (2006), who searched for wide companions of Hipparcos stars in the rNLTT and LSPM catalogs, respectively. Other applications listed in $\S 1$ apply this same principle to get ages, metallicities, etc.

Nevertheless, two of the most exciting applications of wide binaries are the result of statistical studies for which not only the large size but, perhaps most importantly, the 
completeness of any sample of these objects is critical. In particular, their distribution of (physical or angular) separations can be used to gain insights into the processes of star formation, and also to place strong limits to the mass and density of compact dark matter (MACHOs) in the Galactic halo.

Using the rNLTT, Chanamé \& Gould (2004) were able to perform the first comparison of the distributions of separations of large and clean samples of disk and halo wide binaries, as shown in Figure 2a. Somewhat surprisingly, they found both distributions to be the same within the uncertainties, despite the radically different Galactic environments to which they belong today. This provides a unique insight on the environmental conditions in which star formation proceeded in the early Galaxy, and any successful model of the formation of the Galaxy should be able to reproduce this finding.

Finally, the very wide binaries $(a \gtrsim 0.1 \mathrm{pc})$ are so weakly bound that they can be significantly disturbed, even disrupted, by gravitational interactions as they orbit the Galaxy, including those with giant molecular clouds, large-scale tides, passing stars, as well as massive dark objects (MACHOs). If such encounters were frequent during the lifetime of any given binary, then one would expect the widest systems to be relatively depleted. Early attempts to learn about disk dark matter in the solar neighborhood via wide binaries (Bahcall, Hut \& Tremaine 1985) did not achieve useful conclusions due to the small number of wide binaries available then, as well as the intrinsic complexity of the disk potential (Weinberg, Shapiro \& Wasserman 1987).

The situation in the Galactic halo was easier to model, but an adequate sample of halo wide binaries did not exist until the rNLTT binaries were published. Only then, using detailed Monte Carlo simulations of the evolution of a population of wide binaries immersed in a halo filled with compact objects with various combinations of mass and density, Yoo, Chanamé, \& Gould (2004) were able to exclude a Milky Way halo mostly composed of MACHOs with masses $M>43 \mathrm{M}_{\odot}$ at the standard local halo density and at $95 \%$ confidence level, as shown in Figure $2 \mathrm{~b}$. In this way, halo wide binaries were shown to complement the results of the microlensing campaigns, mostly sensitive to lower masses, and provided us with an alternative experiment for addressing the same key question.

\section{Acknowledgements}

I wish to thank Bill Hartkopf for inviting me to this Symposium, and Roeland van der Marel for his interest and support of my work on wide binaries. Thanks as well to Andy Gould and Inese Ivans for a critical reading of this manuscript, and to Freeke van de Voort for her help and enthusiasm in digging into noisy spectra in the search for new binaries in SDSS. Finally, I acknowledge the support of both the American Astronomical Society and the International Astronomical Union through grants to travel and attend the IAU General Assembly.

\section{References}

Allen, C., Poveda, A. \& Hernández-Alcántara, A. 2007, IAU Symposium 240, these proceedings, 405

Allen, C., Poveda, A., \& Herrera, M. 2000, A\&A, 356, 529

Bahcall, J. N., Hut, P., \& Tremaine, S. 1985, ApJ, 290, 15

Bonanos, A. Z., et al. 2006, ApESSS, 79

Bonfils, X., et al. 2005, A\&AA, 442, 635

Catalán, S., et al. 2007, these proceedings, 380

Chanamé, J. \& Gould, A. 2004, ApJ, 601, 289

Desidera, S., et al. 2004, A\&A, 420, 683

Drake, A.J., Cook, K.H., \& Keller, S.C. 2004, ApJ, 607, L29 
Gould, A., \& Chanamé, J. 2004, ApJS, 150, 455

Gould, A., \& Kollmeier, J. 2004, ApJS, 152, 103

Gould, A., \& Salim, S. 2003, ApJ, 582, 1001

Greaves, J. 2005, Journal of Double Star Observations, 1, 41

Hambly, N. C., et al. 2001, MNRAS, 326, 1315

Jiang, G., et al. 2004, ApJ, 617, 1307

Johnston, K., Oswalt, T., \& Valls-Gabaud, D. 2007, these proceedings (Paster), 429

Lépine, S., \& Shara, M. M. 2005, AJ, 129, 1483

Lépine, S., \& Bongiorno, B. 2005, AJ 133, 889

Luyten, W. J. 1979, 1980, New Luyten Catalog of Stars with Proper Motions Larger than Two Tenths of an Arcsecond (Minneapolis: University of Minnesota Press)

Luyten, W.J. 1940-87, The LDS Catalogue: Double Stars with Common Proper Motion (Minneapolis: University of Minnesota Press)

Martín, E.L., 2002, ApJ, 579, 437

Monteiro, H., Jao, W.-C., Henry, T., Subasavage, J., \& Beaulieu, T. 2006, ApJ, 638, 446

Munn, J. A., et al. 2004, AJ, 127, 3034

Paczyński, B. 1997, The Extragalactic Distance Scale, 273 (astro-ph/9608094)

Pagel, B. 1997, Nucleosynthesis and Chemical Evolution of Galaxies (Cambridge Univ Press)

Patten, B.M., et al. 2006, ApJ 651, 502

Poveda, A., et al. 1994, Revista Mexicana de Astronomía y Astrofísica, 28, 43

Rafferty, T. J., et al. 2001, ASP Conf. 232: The New Era of Wide Field Astronomy, 232, 308

Ribas, I., et al. 2005, ApJ, 635, L37

Salim, S., \& Gould, A. 2002, ApJ, 575, L83

Salim, S. \& Gould, A. 2003, ApJ, 582, 1011

Scholz, R.-D., et al. 2002, ApJ, 565, 539

Scholz, R.-D., et al. 2005, A\&BA, 430, L49

Seifahrt, A., et al. 2005, A\& $A, 440,967$

Silvestri, N., et al. 2001, AJ, 121, 503

Silvestri, N., Hawley, S. L., \& Oswalt, T. D. 2005, AJ, 129, 2428

Smith, J.A., Silvestri, N.M., Oswalt, T.D., et al. 2005, ASP Conf. Ser. 334: $14^{\text {th }}$ European Workshop on White Dwarfs, 334, 127

Weinberg, M.D., Shapiro, S.L., \& Wasserman, I. 1987, ApJ, 312, 367

White, R. J., \& Ghez, A. M. 2001, ApJ, 556, 265

Yoo, J., Chanamé, J., \& Gould, A. 2004, ApJ, 601, 311

Zapatero Osorio, M. R., \& Martín, E. L. 2004, A\&AA, 419, 167

\section{Discussion}

Petr Harmanec: Is there some explanation why metallicity depends on eccentricity?

Chaname: The metallicity of a star does not depend on the eccentricity of its orbit, nor viceversa. It is true, however, that the oldest and most metal-poor stars in the Galaxy are halo stars, and these in general have large eccentricities in comparison with the more nearly circular orbits exhibited by disk stars, which, in turn, have higher metal abundances than halo stars. These are statistical facts applicable to these two distinct stellar populations in general, and should not be used in a star-by-star basis. The plot that showed a correlation between metallicity (as indicated by the UV excess) and the eccentricity of the orbits of the stars around the Galaxy is from the 1962 paper by Eggen, Lynden-Bell, \& Sandage (their Figure 4). What I intended to convey by showing this plot is simply how the interplay between the kinematics and abundances of large numbers of stars can be used to infer things about the history of the Galaxy at large.

NANCY Evans: You should be able to get a distribution of mass ratios. How does this work out? 
Chaname: We did compute these distributions, and they are available in Chaname \& Gould (2004). Although this requires the adoption of distances and then color-magnitude relations (therefore introducing model-dependent assumptions and uncertainties), my recollection is that disk and halo wide binaries are also similar in their distributions of mass-ratio. But please take a look at the paper itself for a thorough discussion on this point.

VAleri MAKarov: Do you see the bifurcation of halo stars and disc stars on the colormagnitude diagram $\left(M_{V}\right.$ vs. $\left.(V-J)\right)$ if you use trigomometric parallaxes instead of reduced proper motions?

Chaname: No, the difference is not as clear as with the reduced proper motions, as shown in the quoted paper. It is a useful property of the reduced proper motion method that it reflects the different kinematics of the halo and the disc. 